\title{
High sensitivity biosensor based on dual-peak LPG sensitised by light cladding etching
}

\author{
Xianfeng Chen ${ }^{*}$, Kaiming Zhou, Lin Zhang, Ian Bennion \\ Photonics Research Group, Aston University, Birmingham, B4 7ET, UK
}

\begin{abstract}
We demonstrate a high sensitivity biosensor by fine tailoring mode dispersion and sensitivity of dual-peak LPGs using light-cladding-etching method. The etched device has been used to detect concentration of Hemoglobin protein in sugar solution, showing a sensitivity as high as $20 \mathrm{~nm} / 1 \%$.
\end{abstract}

Keywords: Long-period grating, optical fibre sensors, chemical etching, biosensor

\section{INTRODUCTION}

Long-period fibre gratings (LPGs), as core to cladding mode coupling devices, have been used as band-rejection filters [1], EDFA gain flatteners [2] and optical sensors [3-6]. In comparison with fibre Bragg gratings, LPGs are much more sensitive to the variation of fibre properties and surrounding medium refractive index (SRI). To date, the majority reported work on LPGs employ typical structures with periods of $300-500 \mu \mathrm{m}$, generating phase match resonances distributed in the range $1200-1600 \mathrm{~nm}$. The response of such LPGs can be modified by reducing cladding size via chemical etching [7-9]. It has also been revealed that there exists a set of dispersion turning points in LPG structures, resulting in, a set of conjugate cladding modes [5] which have been experimentally observed as dual-peak LPGs [10, 11]. The dual-peak feature is visible for LPGs with relative short period $\leq 150 \mu \mathrm{m}$ in the extended wavelength range from $900 \mathrm{~nm}$ to $1700 \mathrm{~nm}[6]$. In close proximity to the dispersion turning point, the dual peaks are ultrasensitive to not just external perturbations but also fibre properties. This provides an effective fine-tuning mechanism to tailor the mode dispersion and sensitivity characteristics of dual-peak LPGs for applications.

In this paper, we report an investigation of spectral responses of dual-peak LPGs with reduced claddings realised by chemical etching using hydrofluoric (HF) acid. We experimentally monitored the evolution characteristics of dual resonant peaks, observing rapid transition of the dual-peak features from high order modes to lower ones. A light cladding etching ( $1 \%$ HF concentration) was then performed to finely tailor the sensitivity of the dual-peak LPG.. This etched device was used to measure Hemoglobin protein concentrations in sugar solutions, showing a detection sensitivity as high as $20 \mathrm{~nm} / 1 \%$.

\section{EXPERIMENT}

\subsection{Spectral evolution of dual-peak LPGs with cladding etching}

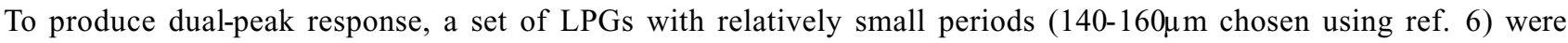
UV-inscribed in hydrogen-loaded standard single mode fibre employing the point-by-point fabrication method and a CW frequency-doubled Ar laser. All the gratings were annealed at $80^{\circ} \mathrm{C}$ for $48 \mathrm{hr}$ to stabilise their optical property. Prior the etching, the spectra of these gratings were measured using a broadband light source and an optical spectrum analyser. Most of these LPGs showed one pair of conjugate resonances companied with a few lower order cladding modes on shorter wavelength side in range $900-1700 \mathrm{~nm}$

In order to effectively control the LPG etching process, we first investigated the etching rate using HF acid of $13 \%$ concentration and non grating-containing fibre samples. Fifteen fibre samples were immersed in the HF solution and were taken out in turn in every $5 \mathrm{~min}$. The samples with different etched claddings were then examined and measured

*chenx2@aston.ac.uk; Fax:+44 (0) 1213590156 
using a microscope with high magnification. Fig. $1 \mathrm{~b}$ plots the cladding radius against etching time, showing an etching rate of $\sim 0.21 \mu \mathrm{m} / \mathrm{min}$. In following, a $20 \mathrm{~mm}$ long dual-peak LPG with a period of $159 \mu \mathrm{m}$ was selected for etching experiment. As the first trace shown in Fig. 1a, this grating has four cladding modes present in the wavelength range from $900 \mathrm{~nm}$ to $1700 \mathrm{~nm}$ and two of which are a pair of conjugate modes located at $1214.9 \mathrm{~nm}$ and $1634.4 \mathrm{~nm}$. Fro m our simulation, we identified them as $L P_{010}, L P_{011}$, and $L P_{012}$ and $L P_{012}^{\prime}$. When this grating was immersed in the HF acid, we monitored the spectrum in-situ and saw clearly a transition of generation, coalescence and annihilation of the dual-peak feature from higher order modes to lower ones. Fig.1a shows the spectral evolution of this grating for the etching period of 67 minutes. From the figure a repeating transition pattern is apparent with reducing cladding size : firstly, the dual peaks ( $L P_{012}$ and $L P_{012}^{\prime}$ ) were moving towards each other, coalesced and eventually annihilated and a new pair of conjugate modes $\left(L P_{011}\right.$ and $L P_{011}^{\prime}$ ) was generated companied by the red-shift of $L P_{010}$; then this transition was repeated leading to the appearance of paired $L P_{010}$ and $L P_{010}^{\prime}$, and $L P_{09}$ modes.

Fig. 1c plots the experimentally measured wavelength shifts of the four modes versus the actual cladding size of the fibre. The dual-peak feature is also evident from Fig. $1 \mathrm{c}$ as $L P_{010}, L P_{011}$ and $L P_{012}$ mode curves are all parabolical. The coalescing points of the $\mathrm{m}=12,11,10$ modes occurred at $1387.1 \mathrm{~nm}, 1385.7 \mathrm{~nm}$ and $1389.1 \mathrm{~nm}$, corresponding to cladding radii of $61.8 \mu \mathrm{m}, 55.9 \mu \mathrm{m}$ and $48.5 \mu \mathrm{m}$, respectively. It is also noticed that the movements of the dual peaks are not linear and symmetric: the loss peak with longer wavelength moves faster than its counterpart. For example, the shift rates of each dual resonant peaks of $\mathrm{m}=11$ mode are $+88.7 \mathrm{~nm} / \mu \mathrm{m}$ and $-156.3 \mathrm{~nm} / \mu \mathrm{m}$ when the cladding reduced from $57.1 \mu \mathrm{m}$ to $56.6 \mu \mathrm{m}$, increasing to $+240.5 \mathrm{~nm} / \mu \mathrm{m}$ and $-292.3 \mathrm{~nm} / \mu \mathrm{m}$ for further cladding reduction from $56.6 \mu \mathrm{m}$ to 55.9 $\mu \mathrm{m}$. This nonlinear behaviour shows that the magnitude change of waveguide dispersion for each mode increases rapidly for wavelengths near the dispersion turning point. The simulation results, shown by lines in Fig.1c, agree quite well with experiment, especially for $L P_{011}$ and $L P_{012}$.
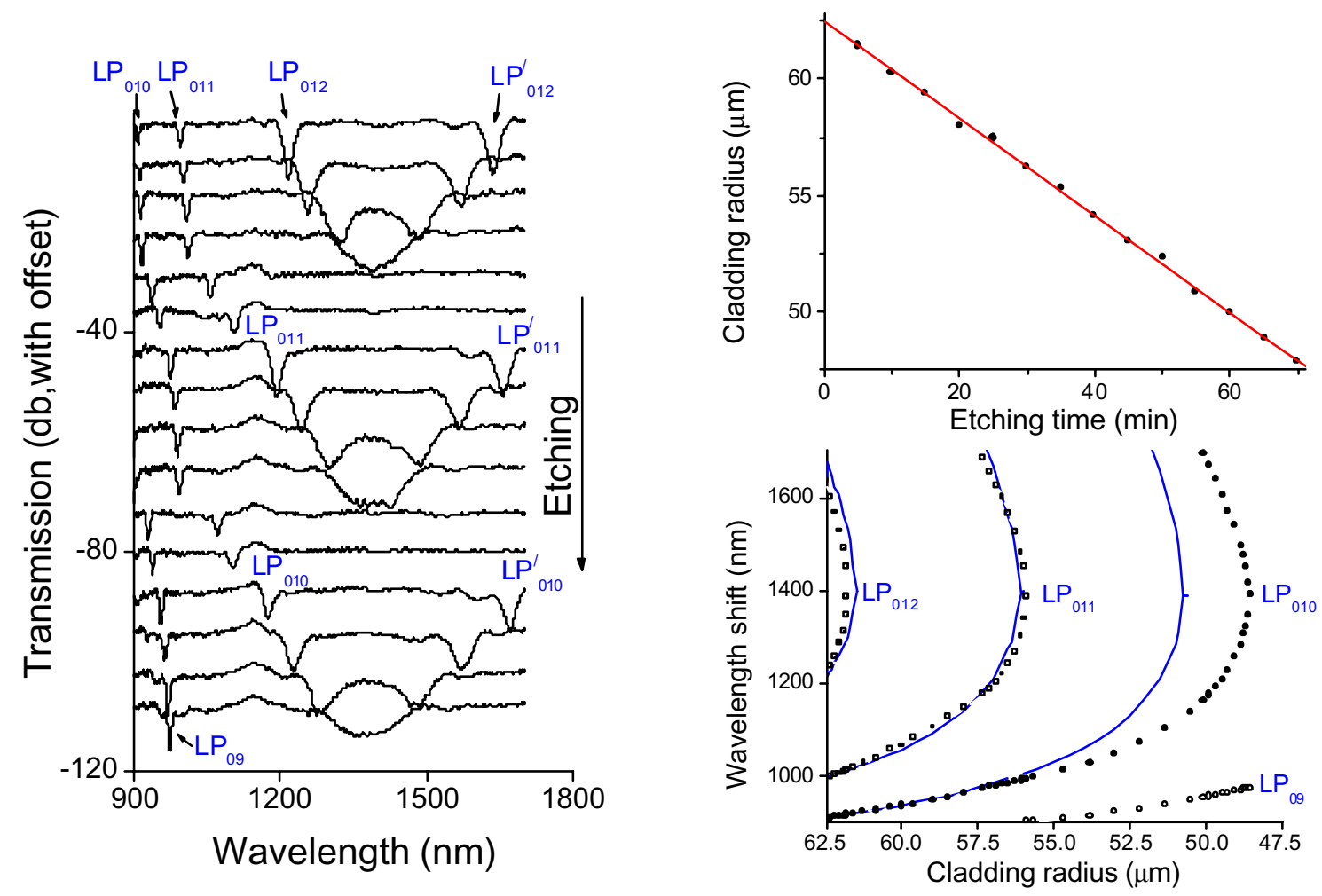

Fig.1 (a) The spectral evolution of a dual-peak LPG of 159- m period during 67min etching process; (b) The cladding radius against etching time; (c). The simulation (line) and experimental (symbol) results of resonance wavelength shift against cladding radius. 


\subsection{Light cladding etching and SRI sensitivity characterisation}

From above experiment we can see that the mode dispersion of dual-peak LPG is ultrasensitive to the cladding size. When the peaks are close to the dispersion turning point, $\sim 1 \mu$ m reduction of the cladding will result in $\sim 250 \mathrm{~nm}$ shift of the resonance. In order to finely control the mode dispersion and sensitivity of dual-peak LPGs, we performed a light etching experiment using HF acid of only $1 \%$ concentration. In this experiment, a 20mm-long LPG with $147 \mu \mathrm{m}$ period was subjected to etching for 96.5 minutes, removing cladding thickness only by $0.9 \mu \mathrm{m}$ (from $62.5 \mu \mathrm{m}$ to $61.4 \mu \mathrm{m}$, measured by the microscope). During the etching process, we also monitored the dual peak evolution in-situ and Fig. $2 \mathrm{a}$ plots the shifts of the dual peaks. The dual peaks were originally at $\mathrm{M}$ and $\mathrm{M}^{\prime}$ spaced by $493.6 \mathrm{~nm}$ and finally moved to $\mathrm{N}$ and $\mathrm{N}^{\prime}$ separated by only $98.1 \mathrm{~nm}$. The sensitivity of this device was significantly enhanced by the light etching as the two peaks were in a much more close proximity to the dispersion tuning point.

The SRI sensitivity of the lightly etched dual-peak LPG was then investigated comparatively with an unetched device of the same grating parameters. The two gratings were immersed in a set of gels with refractive index ranging from 1 to 1.44 and the separation of the dual peaks was measured for each SRI value, as plotted in Fig. 2b. Clearly, the separation increases nonlinearly with increasing SRI, however, that is far larger for the lightly-etched device than for the non-etched one. For SRI vary ing from 1 to 1.44 , the total separation between $N_{\text {and }}^{\prime}$ is $373.9 \mathrm{~nm}$, whereas that between $\mathrm{M}^{\prime}$ and $\mathrm{M}^{\prime}$ is $185.4 \mathrm{~nm}$, only half of the former. This experiment clearly demonstrates that with appropriate etching, the dispersion property of LPG can be finely tailored to bring the dual peaks close to the dispersion turning point, realising ultrahigh SRI sensitivity devices.
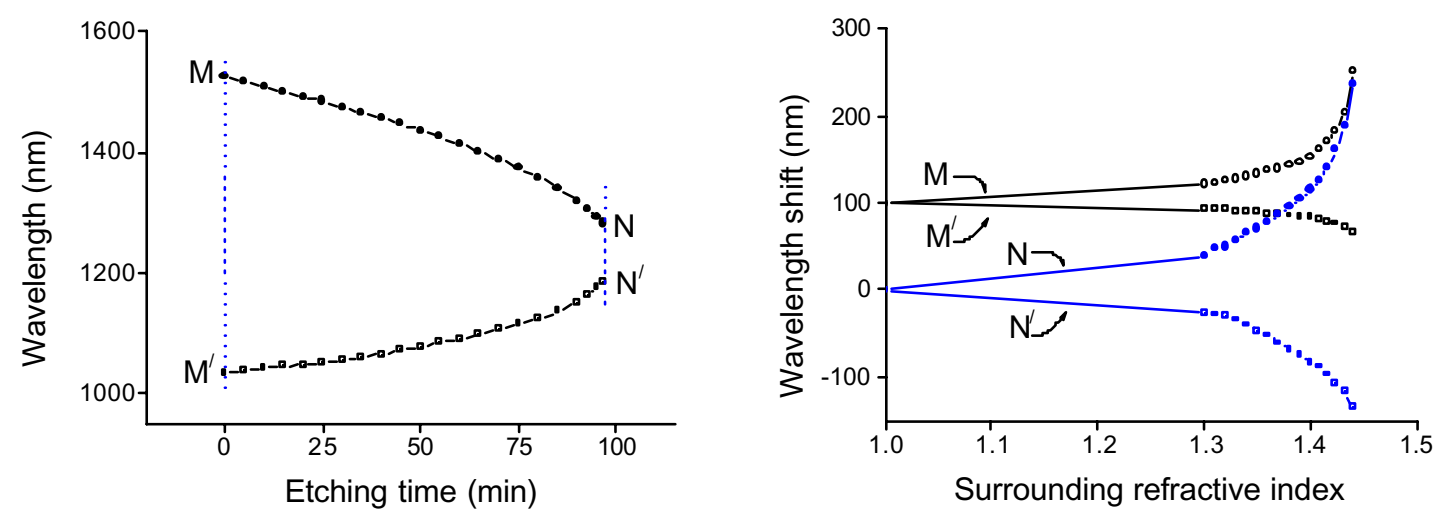

Fig. 2 (a) Dual peak wavelengths against etching time, showing the movement towards the dispersion tuning point; (b) SRI induced spectral separation of the dual peaks for etched (N, $\left.\mathrm{N}^{\prime}\right)$ and unetched (M, $\mathrm{M}$ ') gratings (note: the curves have been offset).

\section{APPICATION}

The lightly-etching dual-peak LPG was used to measure the concentration of the Hemoglobin in sugar solution. Firstly, we prepared a set of Hemoglobin solutions with concentrations from $0.0 \%$ to $1.0 \%$ (step $0.2 \%$ ) by adding Hemoglobin into water. Then $5 \mathrm{ml}$ each of these solutions was drawn and added into six beakers, each beaker had $30 \mathrm{~g}$ of $60 \%$ aqueous sugar solution. We then submerged the lightly-etched dual-peak LPG to these solutions in turn and measured the shifts of N-peak.

Fig. 3a shows the spectra of the N-peak under different solutions and Fig. $3 b$ plots its central wavelength shift against Hemoglobin concentration. We can see when the Hemoglobin concentration changes from $0.0 \%$ to $1.0 \%$, the peak red-shifts by $19.8 \mathrm{~nm}$ Defining the concentration sensitivity as the shift induced by $1 \%$ Hemoglobin, we have a device sensitivity of $\sim 20 \mathrm{~nm} / 1 \%$. Thus, using a standard interrogation system with an optical resolution of $0.1 \mathrm{~nm}$, this finely tailored device could detect the Hemoglobin concentration change as small as $0.005 \%$; a remarkably high detection sensitivity. 

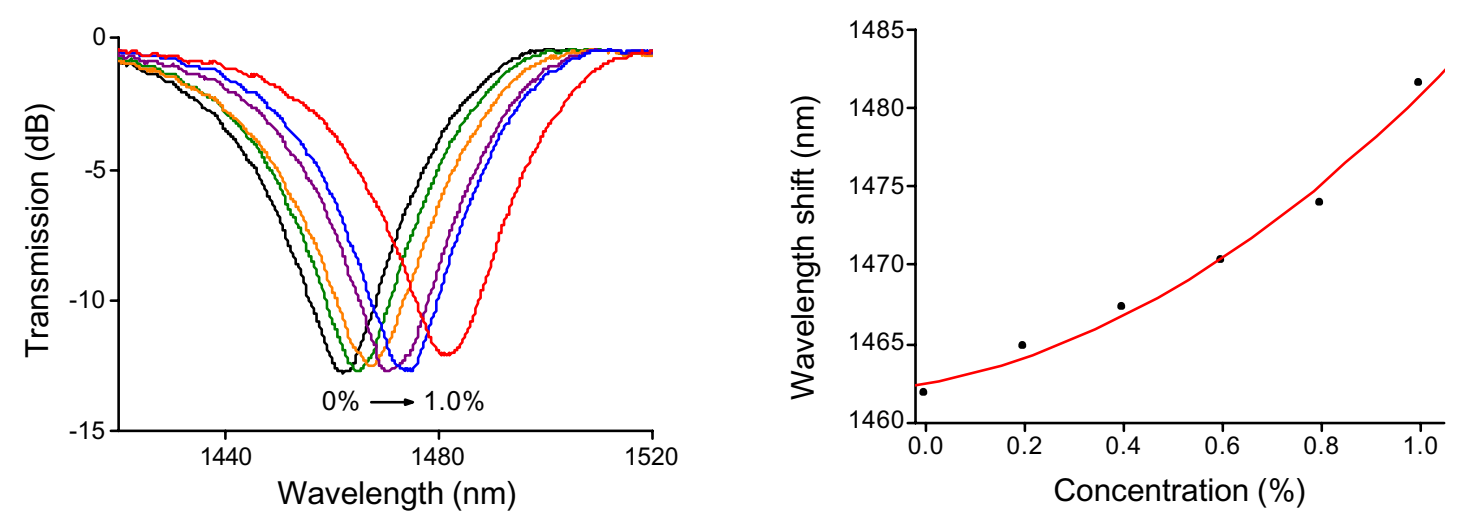

Fig. 3 (a) Spectral evolution of N-peak of the lightly-etched dual-peak LPG with different Hemoglobin concentrations in sugar solutions; (b) N-peak shift against Hemoglobin concentration.

\section{CONCLUSIONS}

We have studied the mode dispersion and sensitivity characteristics of tailored dual-peak LPGs by cladding etching using high- and low-concentration HF acids. With decreasing cladding size, a transition of generation, coalescence and annihilation of dual-peak resonances from higher order modes to lower ones are revealed. The results clearly show that the mode dispersion of the dual-peak LPG is ultrasensitive to the cladding size and thus light-cladding-etching can effectively enhance or finely tailor the sensitivity of the devices. As an implementation of an optical biosensor, we have used the lightly-etched dual-peak LPG to measure Hemoglobin concentrations in sugar buffer solution, demonstrating a concentration sensitivity of $\sim 20 \mathrm{~nm} / 1 \%$. This value indicates that this device has a potential capability to detect protein concentration change as small as $0.005 \%$, which should be enormously attractive for biochemical, medical and environmental sensing applications.

\section{REFERENCES}

[1] A. M. Vengsarkar, P. J. Lemaire, J. B. Judkins, V. Bhatia, T. Erdogan, and J. E. Sipe, "Long-period fiber gratings as band-rejection filters", J. Lightwave Technol., 14, 58-64, 1996.

[2] A. M. Vengsarkar, J. R. Pedrezzani, J. B. Judkins, P. J. Lemaire, N. S. Bergano, and C. R. Davidson, “ Long-period fiber grating-based gain equalizers", Opt. Lett. 21, 336-338, 1996.

[3] V. Bhatia and A. M. Vengsarkar, “ Optical fiber long-period grating sensors”, Opt. Lett. 21, 692-694, 1996.

[4] H. J. Patrick, A. D. Kersey, and F. Bucholtz, "Analysis of the response of long period fiber gratings to external index of refraction", J. Lightwave Technol. 16, 1606-1611, 1998.

[5] X. Shu, L. Zhang, and I. Bennion, “ Sensitivity characteristics near the dispersion turning points of long-period fiber gratings in B/Ge codoped fiber”, Opt. Lett, 26, 1755-1757, 2001.

[6] X. Shu, L. Zhang, and I. Bennion, " Sensitivity characteristics of Long-period fiber gratings", J. Lightwave Technol., 20, 255-266, 2002.

[7] S. Kim, Y. Jeong, S. Kim, J. Kwon, N. Park, and B. Lee, " Control of the characteristics of a long-period grating by cladding etching”, Appl. Opt., 39, 2038-2042, 2000.

[8] K. S. Chiang, Y. Liu, M. N. Ng, and X. Dong, "Analysis of etched long-period fiber grating and its response to external refractive index", Electron. Lett., 36, 966-967, 2000.

[9] X. Chen, K. Zhou, L. Zhang, and I. Bennion, "Optical chemsensors utilizing long-period fiber gratings UV-inscribed in D-fiber with enhanced sensitivity through cladding etching”, IEEE Photon. Technol. Lett. 16, 1352-1354, 2004.

[10] X. Shu, X. Zhu, Q. Wang, S. Jiang, W. Shi, Z. Huang, and D. Huang, “ Dual resonant peaks of LP015 cladding mode in long-period gratings", Electron. Lett., 15, 649-651, 1999.

[11] X. Shu, X. Zhu, S. Jiang, W. Shi, and D. Huang, "High sensitivity of dual resona nt peaks of long-period fibre grating to surrounding refractive index changes", Electron. Lett., 35, 1580-1581, 1999. 